\title{
Semi-implicit two-step hybrid method with FSAL property for solving second-order ordinary differential equations
}

\author{
Nur Azila Yahya* \\ Faculty of Computer and Mathematical Sciences, Universiti Teknologi MARA, Perak Branch, Tapah Campus, 35400 Tapah Road, \\ Perak, Malaysia
}

\section{A R T I C L E IN F O}

\section{Article history:}

Received 3 March 2017

Received in revised form

16 May 2017

Accepted 27 May 2017

\section{Keywords:}

Hybrid method

Oscillatory solution

Interval periodicity

Interval of absolute stability

\section{Introduction}

In this paper we are interested in the numerical solution of initial value problems (IVPs) associated with special second-order ordinary differential equation (ODE) of the forms (Eq. 1)

$y^{\prime \prime}=f(x, y), \quad y(0)=y_{a}, \quad y^{\prime}(0)=y_{b}$.

This problem does not incorporate the first derivative in $f(x, y)$ and the solution relates to oscillatory and periodic solutions. This type of problem commonly arises in the fields of applied sciences such as motion of planet in celestial mechanics, orbital problems, quantum mechanics and electronic. Since most differential equations of celestial mechanics take the form $y^{\prime \prime}=f(x, y)$, it is not surprising that the first attempts at developing methods for Eq. 1 were made by astronomers (Hairer et al., 1993). In recent years, the special second-order ODEs have been extensively studied by many researchers for solving IVPs relates to oscillatory and periodic problems. There has been many research on multistep methods done for Eq. 1, particularly the two-step hybrid method (HM) (Tsitouras, 2003; Coleman, 2003; Franco, 2006; Fang and $\mathrm{Wu}, 2008$; Samat et al., 2012; Ahmat et al., 2013; Jikantora et al., 2015; Franco et al., 2014; Franco and Randez, 2016; Kalogiratou et al., 2016). In a previous work, Coleman (2003), he has investigated the order

\section{* Corresponding Author.}

Email Address: Nurazila7050@perak.uitm.edu.my https://doi.org/10.21833/ijaas.2017.06.024

2313-626X/C) 2017 The Authors. Published by IASE. This is an open access article under the CC BY-NC-ND license

(http://creativecommons.org/licenses/by-nc-nd/4.0/) condition of two-step hybrid method based on the theory of B-series. He discussed these order conditions for general class of two-step hybrid method for problem Eq. 1.

A new class of explicit two-step hybrid method (EHM) which requires less number of stages per step has been developed by Franco (2006). He has considered EHM of order four up to order six. The study by Ahmad et al. (2013) developed semiimplicit two-step hybrid method up to algebraic order five for solving oscillatory problems by taking dispersion relation and solving them together with algebraic conditions of the methods. Later, another study carried out by Jikantora (2015) also developed semi-implicit two-step hybrid method with fifth algebraic order with dispersion and dissipation of higher order.

In this paper, we constructed semi-implicit twostep hybrid method with algebraic order five and six with FSAL feature. The FSAL feature specifies that, the last row of coefficient matrix is same with the vector of output value coefficients. The interval of stability for new methods are also presented and followed by numerical experiments on second-order differential equation for oscillatory or periodic problems. An s-stage two-step hybrid method generally given by (Eqs. 2 and 3)

$Y_{i}=\left(1+c_{i}\right) y_{n}-c_{i} y_{n-1}+h^{2} \sum_{j=1}^{s} a_{i j} f\left(t_{n}+c_{j} h, Y_{j}\right)$

$y_{n+1}=2 y_{n}-y_{n-1}+h^{2}\left[b_{1} f_{n-1}+b_{2} f_{n}+\sum_{i=3}^{s} b_{i} f\left(t_{n}+\right.\right.$

$\left.\left.c_{i} h, Y_{i}\right)\right]$

$i=1, \ldots, s$

The method consists of coefficients which is called the generating matrix, the vector output and 
the vector abscissa which can be represented in Butcher tableau as shown in Table 1.

Table 1: The coefficients of two-step hybrid method

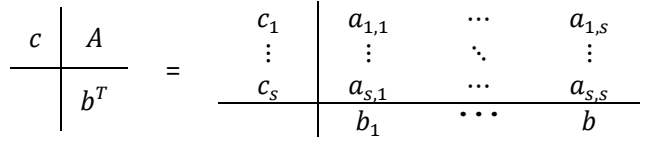

\section{Preliminaries}

The method of the form Eq. 2 and Eq. 3 can be defined as Eqs. 4, 5, and 6.

$Y_{i}=y_{n-1}, \quad Y_{2}=y_{n}$,

$Y_{i}=\left(1+c_{3}\right)-c_{3} y_{n-1}+h^{2} \sum_{j=1}^{i} a_{i j} f\left(t_{n}+c_{j} h, Y_{j}\right)$

$=3, \ldots, s$

$y_{n+1}=2 y_{n}-y_{n-1}+h^{2}\left[\begin{array}{c}b_{1} f_{n-1}+b_{2} f_{n}+ \\ \sum_{i=3}^{s} b_{i} f\left(t_{n}+c_{i} h, Y_{i}\right)\end{array}\right]$

where, $h_{n}=t_{n+1}-t_{n}$ is the step size while $f_{n-1}$ and $f_{n}$ represent approximations for $f\left(t_{n-1}, y_{n-1}\right)$ and $f\left(t_{n}, y_{n}\right)$ respectively. The method only requires to evaluate $s-1$ function evaluation namely $f\left(t_{n}, y_{n}\right), f\left(t_{n}+c_{3} h, Y_{3}\right), \ldots, f\left(t_{n}+c_{s} h, Y_{s}\right)$ in each step. Therefore, this method is considered as twostep hybrid method with $s-1$ stages per step. The tableau of semi-implicit two-step hybrid method with FSAL (SIHMF) feature is as in Table 2

Table 2: The coefficients of SIHMF

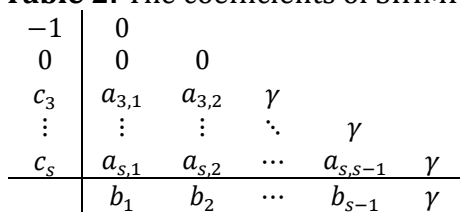

The diagonal elements $a_{33}, a_{44} \ldots, a_{s s}$ in Table 2 are denoted by $\gamma$. The vector output $b^{T}$ corresponding to the output approximation is identical to the last row of $A$. The s-stage implicit two-step hybrid method parameters are given by

$c_{s}=1, a_{s j}=b_{i}$

where, $j=1, \ldots, s$.

In this case, FSAL specifies the s-stage to be the same as the first-stage at the next step given by

$f_{s}=f\left(x_{n+1}, y_{n+1}\right)=f\left(x_{n}+c_{s} h,\left(1+c_{s}\right) y_{n}-y_{n-1}+\right.$ $\left.\sum_{i=1}^{s} a_{s j} f_{j}\right)=f\left(x_{n}+h, 2 y_{n}-y_{n-1}+\sum_{i=1}^{s} b_{i} f_{i}\right)$.

In order to investigate the phase property of twostep hybrid method for solving initial value problem Eq. 1 we consider the second order linear test equation as proposed by Franco (2006) (Eq. 7)

$y^{\prime \prime}=-\lambda^{2}(t)$

If Eq. 2 and Eq. 3 are applied to the test problem Eq. 7, hence it can be written in the vector form as Eqs. 8 and 9

$$
Y=(e+c) y_{n}-c y_{n-1}-H^{2} A Y \quad H=\lambda h,
$$

$y_{n+1}=2 y_{n}-y_{n-1}-H^{2} b^{T} Y$,

where $Y=\left(Y_{1}, \ldots, Y_{s}\right)^{T}, c=\left(c_{1}, \ldots, c_{s}\right)^{T}$ and $e=$ $(1, \ldots, 1)^{T}$. Solving equation in Eq. 8 we obtain Eq. 10

$Y=(e+c) y_{n}\left(I+H^{2} A\right)^{-1}-c y_{n-1}\left(I+H^{2} A\right)^{-1}$,

where

$$
\begin{aligned}
& \left(I+H^{2} A\right)^{-1}=1-H^{2} A+H^{4} A^{2}=1-H^{2} A+H^{4} A^{2}- \\
& \cdots+(-1)^{S-2} H^{2 S-4} A^{S-2} .
\end{aligned}
$$

Substituting Eq. 10 in Eq. 9, then the following recursion relation is obtained Eq. 11:

$y_{n+1}-S\left(H^{2}\right) y_{n}+P\left(H^{2}\right) y_{n-1}=0$,

where

$S\left(H^{2}\right)=2-H^{2} b^{T}\left(I+H^{2} A\right)^{-1}(e+c)$ and

$P\left(H^{2}\right)=1-H^{2} b^{T}\left(I+H^{2} A\right)^{-1} c$

use $S\left(H^{2}\right)$, and $P\left(H^{2}\right)$ to define dispersion error and dissipation error.

Definition 1: The quantities $\varphi(H)$ and $d(H)$ are called the dispersion error (or phase-error) and dissipation error, respectively (Eq. 12)

$\varphi(H)=H-\arccos \left(\frac{S\left(H^{2}\right)}{2 \sqrt{\left(H^{2}\right)}}\right)$
$d(H)=1-\sqrt{P\left(H^{2}\right)}$.

According to Simos et al. (2003), the dispersion is the angle between the true and the approximate solution and the dissipation is the distance from a standard cyclic solution. The method is said to be dispersive of order $q$ and dissipative of order $r$, if

$\varphi(H)=\left(H^{q+1}\right) \quad d(H)=\left(H^{r+1}\right)$

the stability of two-step hybrid method will be calculated using the interval of periodicity and interval of absolute stability which are determined by characteristic polynomial.

Definition 2: The polynomial (Eq. 13)

$\xi^{2}-S\left(H^{2}\right) \xi+P\left(H^{2}\right)=0$

is called characteristic polynomial of Eq. 11. Twostep hybrid method has periodicity interval if coefficient of Eq. 13 satisfy the condition (Eq. 14)

$P\left(H^{2}\right) \equiv 1, \quad\left|S\left(H^{2}\right)\right|<2, \quad \forall H \in\left(0, H_{P}\right)$

The method satisfies the condition Eq. 14 are called zero dissipative $(d(H)=0)$. When the methods have a finite order of dissipation, means the interval of periodicity is $(0, \propto)$, the integration process is stable or remains bounded if the coefficient Eq. 13 satisfy the conditions (Eq. 15)

$\left|P\left(H^{2}\right)\right|<1$, 
and

$\left|S\left(H^{2}\right)\right|<1+P\left(H^{2}\right)$

$\forall H \in\left(0, H_{S}\right)$

The two-step hybrid method are derived by using order conditions which are the set of simultaneous equations which contain the coefficients. The solution of simultaneous equations gives the value of coefficients in terms of the free parameter associated to the local truncation error $e_{p+1}$. The coefficients are then substituted into the error constant $E_{p+1}$.

The minimized value of the free parameter is obtained by optimizing the error constant with respect to the free parameter. The $p$ th-order error constant is a quantity defined by

$$
\begin{aligned}
& E_{p+1}=\left\|e_{p+1}\left(t_{1}\right), \ldots, e_{p+1}\left(t_{k}\right)\right\|_{2} \\
& =\sqrt{e_{p+1}\left(t_{1}\right)^{2}+\cdots+e_{p+1}\left(t_{k}\right)^{2}}
\end{aligned}
$$

where, $k$ is the number of trees of order $p+$ $2\left(\rho\left(t_{i}\right)=\rho+2\right)$ and $e_{p+1}\left(t_{i}\right)$ is local truncation error as defined in Coleman (2003). The order conditions up to algebraic order six given in Coleman (2003) are (Eqs. 17, 18, 19, 20, 21, 22)

Order 2

$\sum b_{i}=0$,

Order 3

$\sum b_{i} c_{i}=0$

Order 4

$\sum b_{i} c_{i}^{2}=\frac{1}{6}$

Order 5

$\sum b_{i} c_{i}^{3}=0, \sum b_{i} a_{i j} c_{j}=0$

Order 6

$\sum b_{i} c_{i}^{4}=\frac{1}{15}, \sum b_{i} c_{i} a_{i j} c_{j}=-\frac{1}{60}$

$\sum b_{i} a_{i j} c_{j}^{2}=\frac{1}{180}$

and together with simplifying condition

$\sum a_{3 j}=\frac{c_{3}^{2}+c_{3}}{2}, \sum a_{4 j}=\frac{c_{4}^{2}+c_{4}}{2}$

\section{Derivation of method SIHM with FSAL property}

Presented in this section is the derivation of semiimplicit two-step hybrid method of order five designed using FSAL property.

\subsection{Fifth order SIHM}

In this case, FSAL specifies the four-stages to be the same as the first-stage at the next step given by

$f_{4}=f\left(x_{n+1}, y_{n+1}\right)=f\left(x_{n}+c_{4} h,\left(1+c_{4}\right) y_{n}-y_{n-1}+\right.$ $\left.\sum_{i=1}^{4} a_{4 j} f_{j}\right)=f\left(x_{n}+h, 2 y_{n}-y_{n-1}+\sum_{i=1}^{4} b_{i} f_{i}\right)$

The fourth-stage two-step hybrid method parameters are given by

$c_{4}=1, a_{4 j}=b_{i}, \quad j=1,2,3,4$
To derive fifth-order SIHMF method, we use the algebraic order conditions Eq. 17, Eq. 20 and Eq. 22. There are six equations and seven unknowns that have to be satisfied giving one free parameter which is chosen to be $b_{3}$. The system of equations are solved simultaneously to obtain the values of coefficients in terms of $b_{3}$ which are given by the expression

$b_{1}=\frac{1}{12}, \quad b_{2}=\frac{5}{6}, \quad c_{3}=1$,

$a_{31}=0, \quad a_{32}=\frac{11}{12}+b_{3}, \quad a_{33}=-b_{3}+\frac{1}{12}$

by minimizing constant error in Eq. 16 we have

$b_{3}=0$

and

$E_{5}=3.0619 \times 10^{-2}$

This method is denoted as SIHMF5 and can be expressed diagrammatically as in Table 3 . The interval of periodicity is given by $(0, \sqrt{6})$.

Table 3: The coefficients of SIHFM of order five

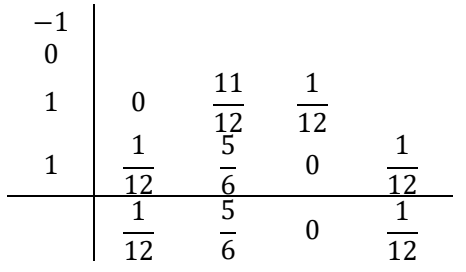

\subsection{Sixth order SIHM}

In this case, FSAL specifies the five-stages to be the same as the first-stage at the next step given by

$f_{5}=f\left(x_{n+1}, y_{n+1}\right)=f\left(x_{n}+c_{5} h,\left(1+c_{5}\right) y_{n}-y_{n-1}+\right.$

$\left.\sum_{i=1}^{5} a_{5 j} f_{j}\right)=f\left(x_{n}+h, 2 y_{n}-y_{n-1}+\sum_{i=1}^{5} b_{i} f_{i}\right)$.

The fifth-stage two-step hybrid method parameters are given by

$c_{5}=1, \quad a_{5 j}=b_{i}, \quad j=1,2,3,4,5$.

To derive the new method, we use the algebraic order conditions Eq. 17 and Eq. 22. There are 10 equations and 12 unknowns that have to be satisfied giving two free parameter which is chosen to be $c_{4}$ and $\gamma$. Solving all conditions simultaneously to obtain the values of coefficients in terms $c_{4}$ and $\gamma$. By minimizing constant error Eq. 4 , we obtain $\gamma=-\frac{1}{40}$ and $c_{4}=\frac{163}{100}$ and $E_{6}=4.4213 \times 10^{-2}$. This method is denoted as SIHMF6 and can be expressed diagrammatically as in Table 4 . The interval of absolute stability is given by $(0,2.18)$.

\section{Results and discussion}

In this section, we present five problems which have oscillatory solution. All the problems will be tested 
by the semi-implicit FSAL methods to evaluate the

effectiveness of new method.

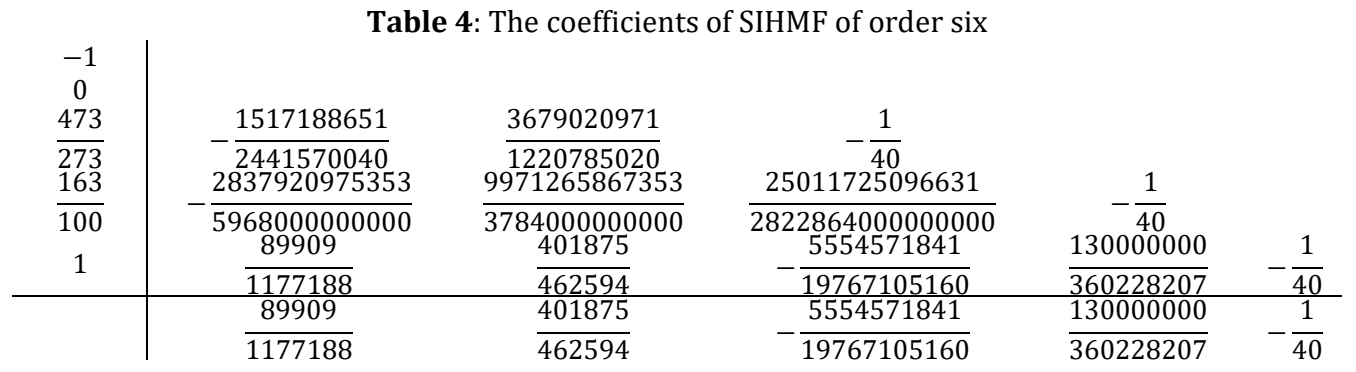

The fifth order method, SIHMF5 is compared with semi-implicit hybrid method of order five with fourstages derived by Ahmad et al. (2013) and explicit two-step hybrid method of order four with threestages derived by Franco (2006). The sixth order method is compared with two other methods derived by Franco (2006).

The methods that have been used in comparisons are denoted by

(i) SIHM4(5): Fifth order semi-implicit method with four-stage derived by Ahmad et al. (2013).

(ii) EHM4: Fourth order explicit hybrid method with four-stage derived by Franco (2006).

(iii) EHM6: Sixth order explicit hybrid method with five-stage derived by Franco (2006).

(iv) EHM5: Fifth order explicit hybrid method with four-stage derived by Franco (2006).

The criterion used in the numerical comparison is decimal logarithm of the maximum error versus step-sizes required by each method.

Absolute error $=$ max error $\left|y\left(t_{n}\right)-y_{n}\right|$

where, $y\left(t_{n}\right)$ is exact solution and $y_{n}$ is approximate solution. The test problems used are listed below:

Problem 1: Homogeneous Problem studied by Franco (2006)

$$
y^{\prime \prime}=-y \quad y(0)=0 \quad y^{\prime}(0)=1
$$

Exact solution is $y=\sin (x)$. The numerical results are shown in Fig. 1 and Fig. 2.

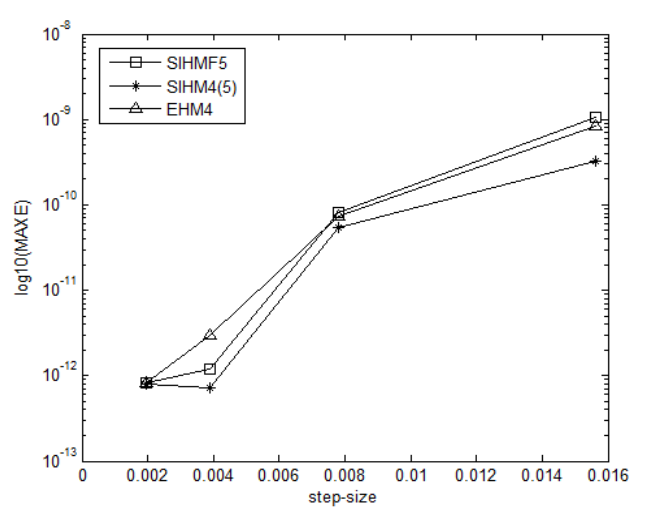

Fig. 1: The efficiency curve for SIHMF5 method for Problem 1 with $h=0.125 / 2^{i}, i=2,3,4,5$

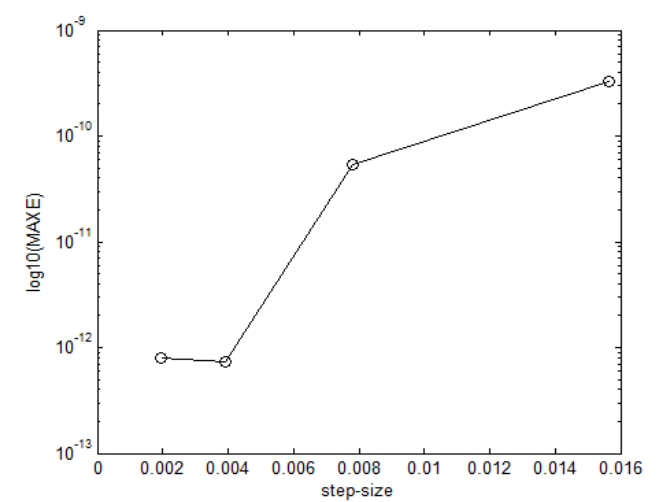

Fig. 2: The efficiency curve for SIHMF6 method for Problem 1 with $h=0.125 / 2^{i}, i=2,3,4,5$

Problem 2: The two-body gravitational problem studied by Dormand et al. (1987)

$$
\begin{array}{ll}
y_{1}^{\prime \prime}=\frac{-y_{1}}{\left(\sqrt{y_{1}^{2}+y_{2}^{2}}\right)^{3}}, & y_{1}(0)=1-e, \quad y_{1}^{\prime}(0)=0, \\
y_{2}^{\prime \prime}=\frac{-y_{2}}{\left(\sqrt{y_{1}^{2}+y_{2}^{2}}\right)^{3}}, & y_{2}(0)=0, \quad y_{2}^{\prime}(0)=\sqrt{\frac{1+e}{1-e}},
\end{array}
$$

with $e$ representing the eccentricity of an orbit. The exact solution is $y_{1}(x)=\cos (E)-e$ and $y_{2}=$ $\sqrt{1-e^{2}} \sin (E)$ with $E$ satisfies the Kepler's equation $x=E-e \sin (E)$. Numerical results is for the case $e=0$. The numerical results are shown in Fig. 3 and Fig. 4.

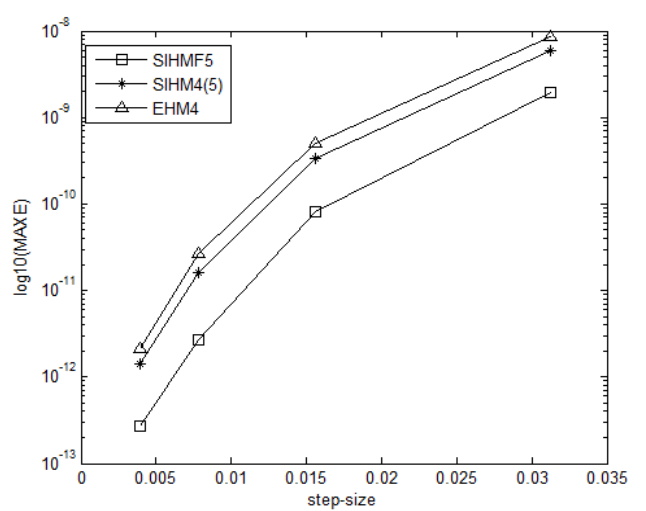

Fig. 3: The efficiency curve for SIHMF5 method for Problem 2 with $h=0.125 / 2^{i}, i=2,3,4,5$

Problem 3: Orbital Problem studied by Van der Houwen and Sommeijer (1989)

$$
\begin{aligned}
& y_{1}^{\prime \prime}=-4 x^{2} y_{1}-\frac{2 y_{2}}{\sqrt{y_{1}^{2}+y_{2}^{2}}}, \quad y_{1}(0)=1, \quad y_{1}^{\prime}(0)=0, \\
& y_{2}^{\prime \prime}=-4 x^{2} y_{2}-\frac{2 y_{1}}{\sqrt{y_{1}^{2}+y_{2}^{2}}}, \quad y_{2}(0)=0, \quad y_{2}^{\prime}(0)=0,
\end{aligned}
$$


exact solution is $y_{1}(x)=\cos ^{2}(x)$ and $y_{2}(x)=$ $\sin ^{2}(x)$. The numerical result are shown in Fig. 5 and Fig. 6.

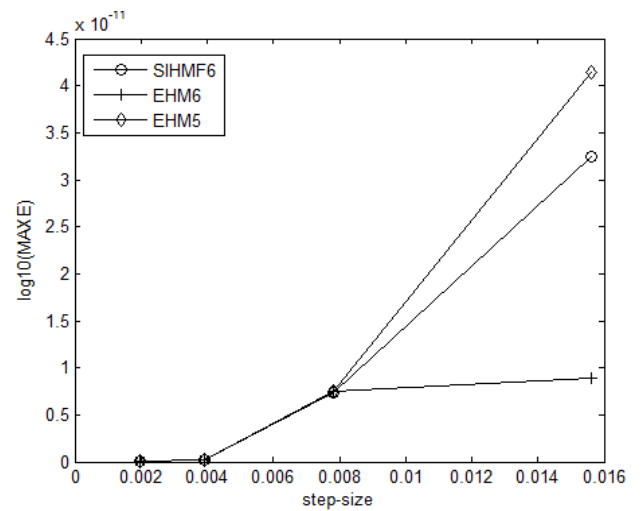

Fig. 4: The efficiency curve for SIHMF6 method for Problem 2 with $h=0.125 / 2^{i}, i=3,4,5,6$

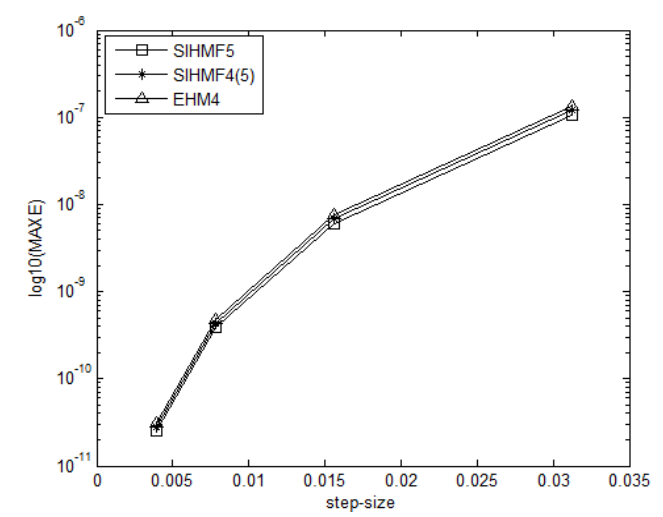

Fig. 5: The efficiency curve for SIHMF5 method for Problem 3 with $h=0.125 / 2^{i}, i=2,3,4,5$

Problem 4: Two-body problem studied by Franco (2003)

$y_{1}^{\prime \prime}+\omega^{2} y_{1}=\frac{2 y_{1} y_{2}-\sin (2 \omega x)}{\sqrt{\left(y_{1}^{2}+y_{2}^{2}\right)^{3}}}, y_{1}(0)=1, y_{1}^{\prime}(0)=0$,

$y_{2}^{\prime \prime}+\omega^{2} y_{2}=\frac{y_{1}-y_{2}-\cos (2 \omega x)}{\sqrt{\left(y_{1}^{2}+y_{2}^{2}\right)^{3}}}, \quad y_{2}(0)=0, \quad y_{2}^{\prime}(0)=\omega$

where $\omega=1$ The exact solution is $y_{1}(x)=\cos (x)$ and $y_{2}(x)=\sin (x)$. The numerical results are shown in Fig. 7 and Fig. 8.

Problem 5: an almost periodic orbit problem given in Stiefel and Bettis (1969)

$y_{1}^{\prime \prime}=-y_{1}+\cos (x), \quad y_{1}(0)=1, \quad y_{1}^{\prime}(0)=0$,

$y_{2}^{\prime \prime}=-y_{2}+\sin (x), \quad y_{2}(0)=0, \quad y_{2}^{\prime}(0)=0.9995$,

exact solution is $y_{1}(x)=\cos (x)+0.0005 x \sin (x)$ and $y_{2}(x)=\sin (x)-0.0005 x \cos (x)$. The numerical results are shown in Fig. 9 and Fig. 10

From Fig. 3, Fig. 5 and Fig. 7, we observed that the new SIHMF5 is performed better compared to SIHM4(5) and EHM4. However in Fig. 1 shows that SIHM4(5) is performed better than SIHMF5 and EHM4. While in Fig. 9 we observed that, all methods have almost equal performance.
From Fig. 2, Fig. 4, Fig. 6, Fig. 8 and Fig. 10 we observed that the new SIHMF6 have almost equal performance with EHM6 and EHM5.

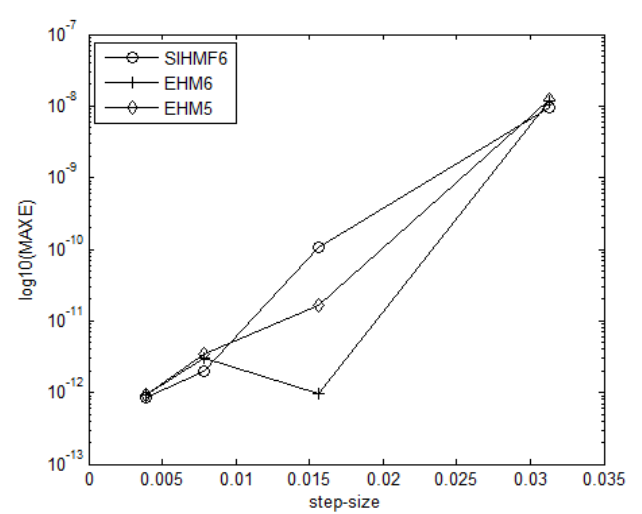

Fig. 6: The efficiency curve for SIHMF6 method for Problem 3 with $h=0.125 / 2^{i}, i=2,3,4,5$

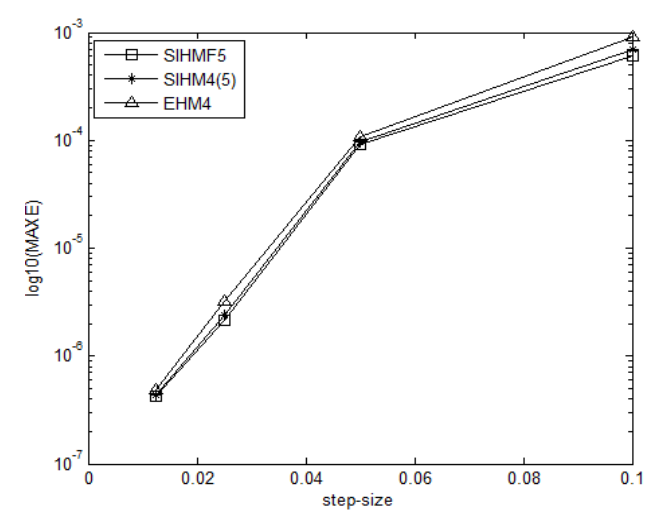

Fig. 7: The efficiency curve for SIHMF5 method for Problem 4 with $h=0.1 / 2^{i}, i=0,1,2,3$

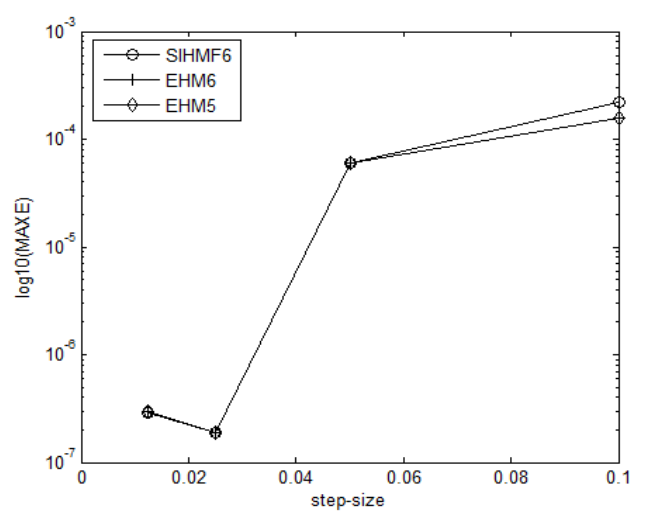

Fig. 8: The efficiency curve for SIHMF6 method for Problem 4 with $h=0.1 / 2^{i}, i=0,1,2,3$

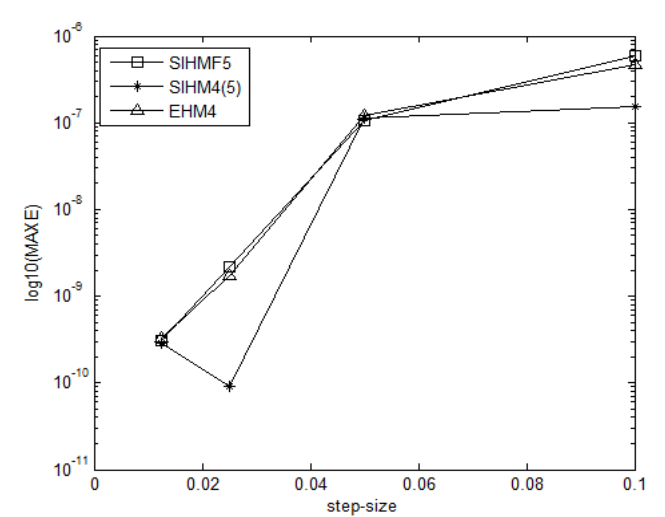

Fig. 9: The efficiency curve for SIHMF5 method for Problem 5 with $h=0.1 / 2^{i}, i=0,1,2,3$ 


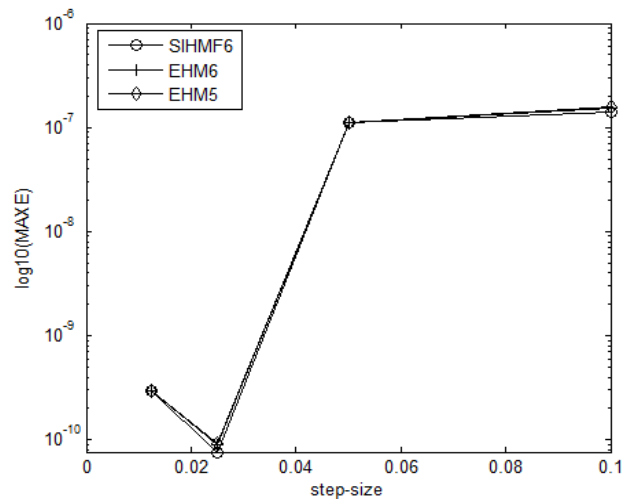

Fig. 10: The efficiency curve for SIHMF6 for Problem 5 with $h=0.1 / 2^{i}, i=0,1,2,3$

\section{Conclusion}

Two semi-implicit two-step hybrid method of order five and six designed using FSAL property for solving second-order IVPs with oscillatory solution are derived. The results of comparison based on maximum error evaluation at different step-sizes were used for comparison purpose as shown in Figs. 1-10. Our new method, SIHMF5 and SIHMF6 can be an alternative method for solving oscillatory problems and can be advantageous to the Science and Technology fields.

\section{References}

Ahmad SZ, Ismail F, and Senu N (2013). Semi implicit hybrid methods with higher order dispersion for solving oscillatory problems. In: Ishak A, Hashim I, Ismail ES, and Nazar R (Eds.) AIP Conference Proceedings, 1522(1): 553-560. https://doi.org/10.1063/1.4801174

Coleman JP (2003). Order condition for a class of two-step hybrid methods for $y^{\prime \prime}=f(x, y)$. IMA Journal of Numerical Analysis, 23(2): $197-220$.

Dormand JR, El-Mikkawy MEA, and Prince PJ (1987). Families of Runge-Kutta-Nystrom formulae. IMA Journal Numerical Analysis, 7(2): 235-250.
Fang Y and Wu X (2008). A trigonometrically fitted explicit numerov-type method for second order initial value problems with oscillating solution. Applied Numerical Mathematics, 58(3): 341-351.

Franco JM (2003). A 5 (3) pair of explicit ARKN methods for the numerical integration of perturbed oscillators. Journal of Computational and Applied Mathematics, 161(2): 283-293.

Franco JM (2006). A class of two-step hybrid methods for secondorder IVPs. Journal of Computational and Applied Mathematis, 187(1): 41-57.

Franco JM, Gómez I, and Rández L (2014). Optimization of Explicit two-step hybrid methods for solving orbital and oscillatory Problems. Computer Physics Communications, 185(10): 25272537.

Franco JM, Rández L (2016). Explicit exponentially fitted two-step hybrid method of high order for second-order oscillatory IVPs. Applied Mathmetics and Computation, 273: 493-505.

Hairer E, Norsett SP, and Wanner G (1993). Solving ordinary differential equations 1: Nonstiff Problems. Springer-Verlag Berlin Heidelberg, Berlin, Germany.

Jikantora YD, Ismail F, and Senu N (2015). Zero dissipative semiimplicit hybrid method for solving oscillatory or periodic problems. Applied Mathematics and Computation, 252: 388396.

Kalogiratou Z, Monovasilis T, Higinio Ramos, and Simos TE (2016). A new approach on construction on trigonometrically fitted two-step hybrid methods. Journal of Computational and Applied Mathematics, 303: 146-155.

Samat F, Ismail F, and Suleiman M (2012). High order explicit hybrid methods for solving second order ordinary differential equations. Sains Malaysiana, 41(2): 253-260.

Simos TE, Famelis IT, and Tsitouras C (2003). Zero dissipative, explicit numerov-type methods for second order IVPs with oscillating solutions. Numerical Algorithms, 34(1): 27-40.

Stiefel E and Bettis DG (1969) Stabilization of Cowell's method. Numerische Mathematik, 13(2): 154-175.

Tsitouras C (2003). Families of explicit two-stepmethods for integration of problems with oscillating solutions. Applied Mathematics and Computation, 135(1): 169-178.

Van der Houwen PJ and Sommeijer BP (1989). Diagonally implicit Runge-Kutta-Nystrom methods for oscillatory problems. SIAM Journal on Numerical Analysis, 26(2): 414-429. 